for

\title{
Efficient Plasmon-Mediated Energy Funneling to the Surface of Au@Pt Core-Shell Nanocrystals
}

Christian Engelbrekt ${ }^{1,2, *}$, Kevin Thomas Crampton ${ }^{1}$, Dmitry A. Fishman ${ }^{1}$, Matt Law ${ }^{1}$, Vartkess Ara Apkarian ${ }^{1, *}$

${ }^{1}$ Department of Chemistry, University of California Irvine, Irvine, California, 92697, United States, ${ }^{2}$ Department of Chemistry, Technical University of Denmark, Kongens Lyngby, 2800, Denmark

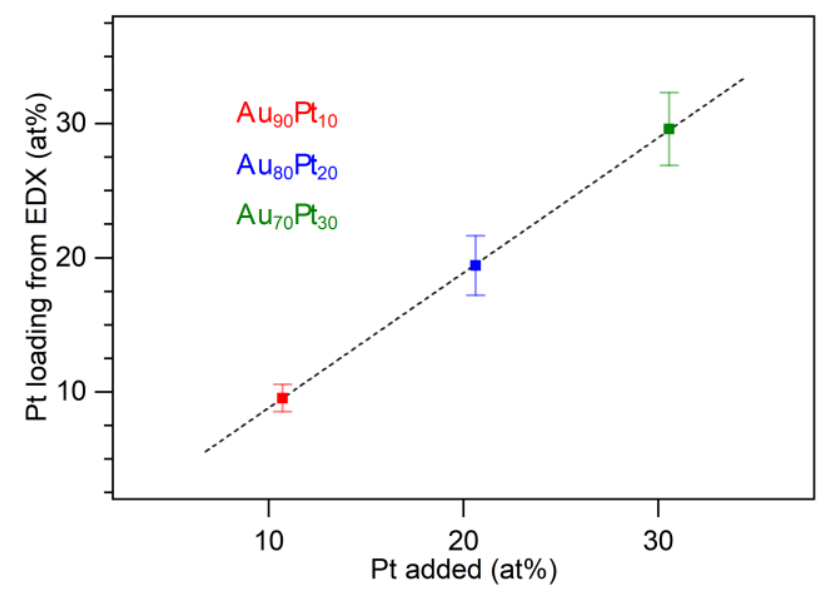

Figure S1. Pt content of the purified Au@Pt NCs as a function of the Pt at $\%$ added during NC synthesis as measured by EDX spectroscopy in the TEM. We find a Pt content of 9.5 $\pm 0.1,19.4 \pm 0.2$ and $29.6 \pm$ 0.3 at $\%$ for 10.7, 20.6, and 30.6 at\% added $\mathrm{Pt}$, respectively. Therefore, we refer to the products as $\mathrm{Au}_{90} \mathrm{Pt}_{10}$, $\mathrm{Au}_{80} \mathrm{Pt}_{20}$, and $\mathrm{Au}_{70} \mathrm{Pt}_{30} \mathrm{NCs}$. 

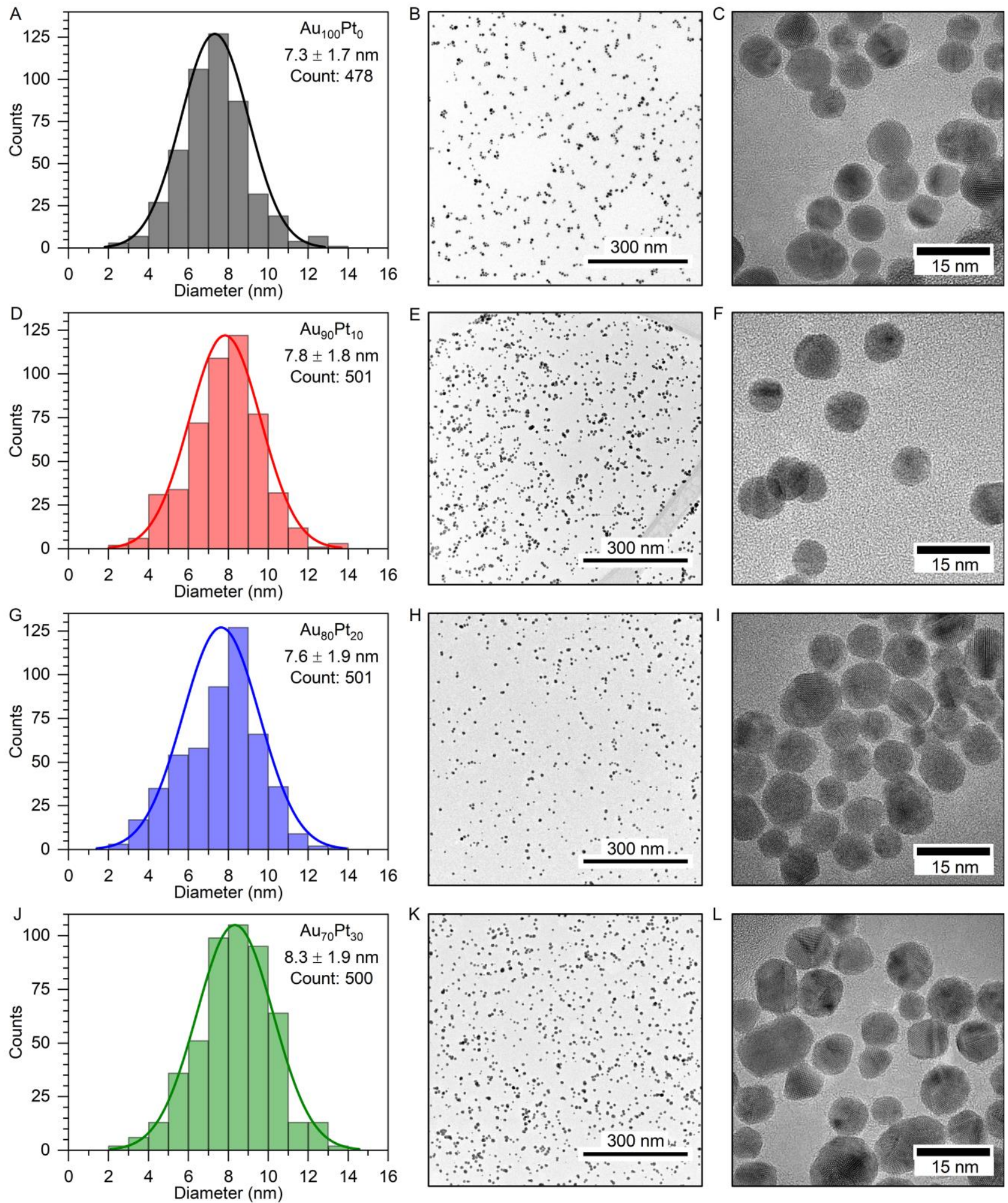

Figure S2. TEM analysis of NC size and shape. (left) Diameter histograms of $500 \mathrm{NCs,} \mathrm{(middle)}$ representative low-magnification TEM images, and (right) representative higher-magnification TEM images of (A-C) $\mathrm{Au}_{100} \mathrm{Pt}_{0}$, (D-F) $\mathrm{Au}_{90} \mathrm{Pt}_{10},(\mathrm{G}-\mathrm{I}) \mathrm{Au}_{80} \mathrm{Pt}_{20}$ and (J-L) $\mathrm{Au}_{70} \mathrm{Pt}_{30} \mathrm{NCs}$. 

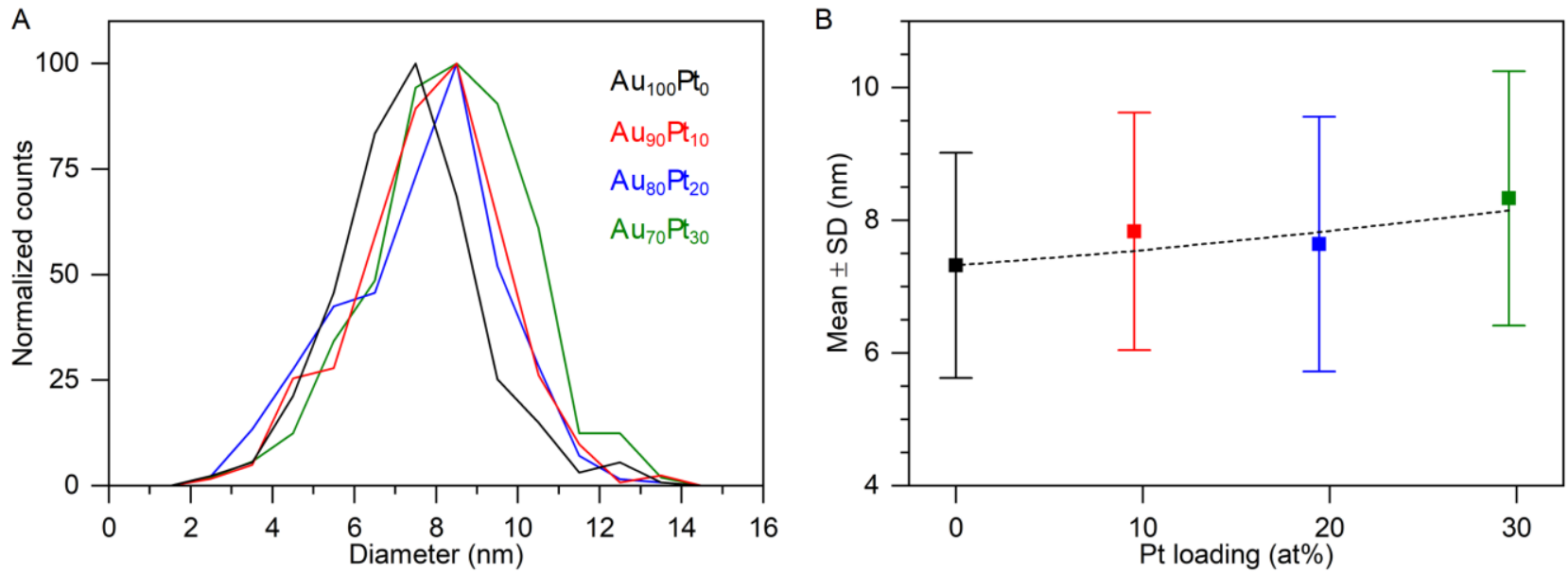

Figure S3. (A) Overlay of the NC diameter histograms in Figure S2. (B) Statistical mean \pm 1 standard deviation of $\mathrm{NC}$ diameters as a function of $\mathrm{Pt}$ loading. The dashed line represents the calculated diameter of a spherical core-shell $\mathrm{NC}$ with a core diameter of $7.32 \mathrm{~nm}$ (as measured for $\mathrm{Au}_{100} \mathrm{Pt}_{0}$ ) and a shell at\% given by the experimentally measured Pt loading. 

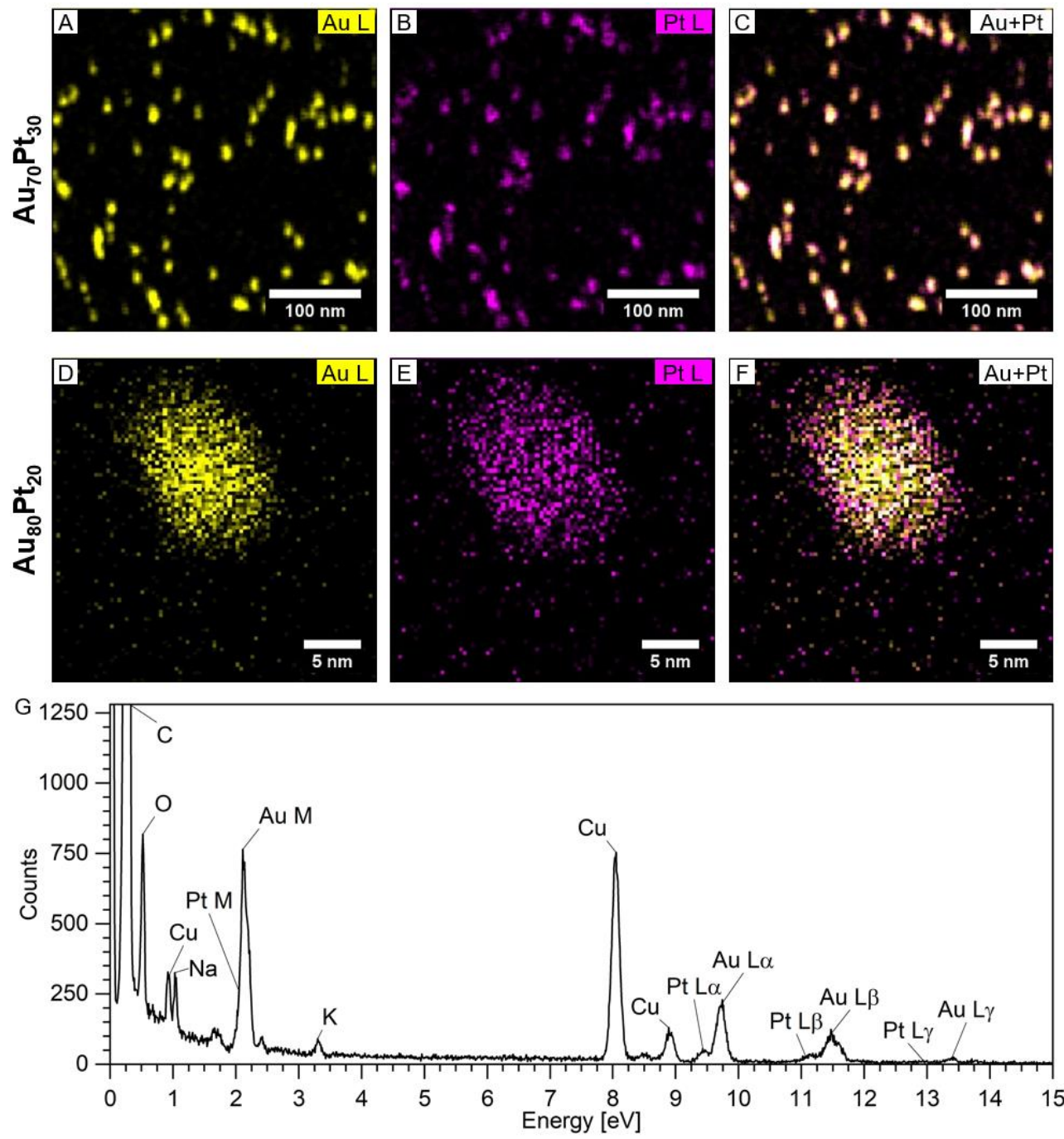

Figure S4. STEM-EDX elemental maps of (A-C) ensemble of $\mathrm{Au}_{70} \mathrm{Pt}_{30} \mathrm{NCs}$, and (D-F) single $\mathrm{Au}_{80} \mathrm{Pt}_{20}$ NC. Yellow (A and D) represents Au and. magenta (B and E) Pt. C and $\mathrm{E}$ are overlays of the $\mathrm{Au}$ and Pt maps. (G) EDX spectrum extracted from the map in D-F.

The shell thickness, $l$, in core-shell particles can alternatively be calculated based on measured wt\% (e.g. from TGA, EDX, ICP-MS or ICP-OES) via this general approach:

$$
\begin{gathered}
l=\overbrace{\left[\frac{3}{4 \pi}\left(V_{\text {core }}+V_{\text {shell }}\right)\right]^{\frac{1}{3}}}^{\text {core@shell radius }}-\frac{1}{2} D_{\text {core }}=\left[\frac{3}{4 \pi} V_{\text {core }}\left(1+\frac{w t \% \rho_{\text {core }}}{(100-w t \%) \rho_{\text {shell }}}\right)\right]^{\frac{1}{3}}-\frac{1}{2} D_{\text {core }}=\left[\frac{3}{4 \pi} \cdot \frac{4 \pi}{3}\left(\frac{1}{2} D_{\text {core }}\right)^{3}\left(1+\frac{w t \% \rho_{\text {core }}}{(100-w t \%) \rho_{\text {sthel }}}\right)\right]^{\frac{1}{3}}-\frac{1}{2} D_{\text {core }} \\
=\left[\left(\frac{1}{2} D_{\text {core }}\right)^{3} \cdot\left(1+\frac{w t \% \rho_{\text {core }}}{(100-w t \%) \rho_{\text {sthel }}}\right)\right]^{\frac{1}{3}}-\frac{1}{2} D_{\text {core }}
\end{gathered}
$$

where $V_{\text {core, }}, V_{\text {shell }}, D_{\text {core }}, \rho_{\text {core }}$, and $\rho_{\text {shell }}$ are the core volume, shell volume, core diameter, core density, and shell density, respectively. For the $\mathrm{Au}_{100} \mathrm{Pt}_{0}, \mathrm{Au}_{90} \mathrm{Pt}_{10}, \mathrm{Au}_{80} \mathrm{Pt}_{20}$, and $\mathrm{Au}_{70} \mathrm{Pt}_{30}, l$ is 0 , $1.1,2.5$ and $4.1 \AA$, respectively. 

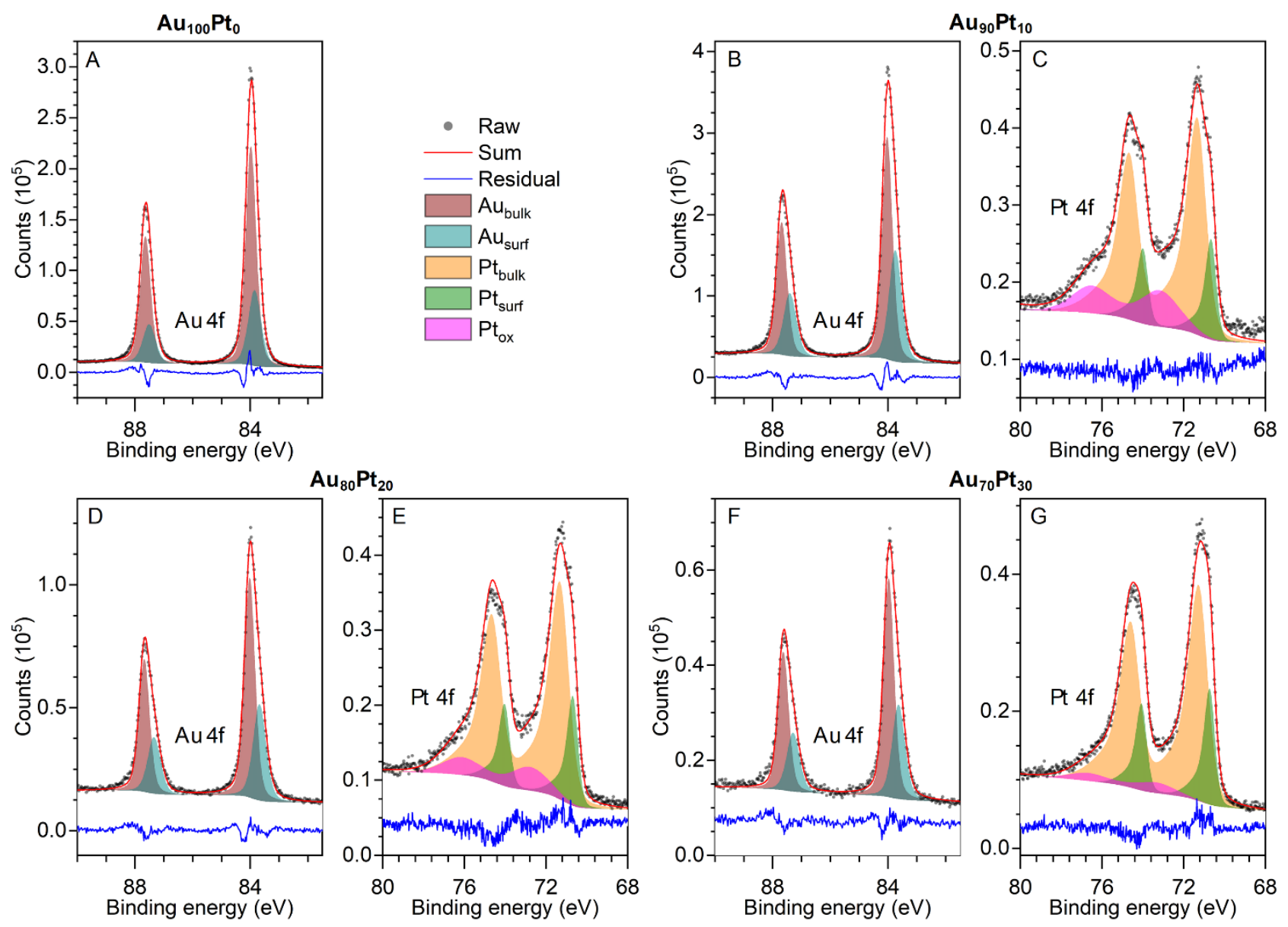

Figure S5. Decomposition of $\mathrm{Au}(4 \mathrm{f})$ and $\mathrm{Pt}(4 \mathrm{f}) \mathrm{PES}$ spectra for $(\mathrm{A}) \mathrm{Au}_{100} \mathrm{Pt}_{0},(\mathrm{~B}-\mathrm{C}) \mathrm{Au}_{90} \mathrm{Pt}_{10}$, (D-E) $\mathrm{Au}_{80} \mathrm{Pt}_{20}$, and $(\mathrm{F}-\mathrm{G}) \mathrm{Au}_{70} \mathrm{Pt}_{30} \mathrm{NCs}$ at $h v=220 \mathrm{eV}$. Gray circles represent the raw data, shaded areas the contributions from components as given in the legend, red lines the fit sum, and blue lines the fit residuals.

Table S1. Fitting parameters from deconvolution of Au(4f) and Pt(4f) PES spectra as shown in Figure S5.

\begin{tabular}{|c|c|c|c|c|c|c|c|c|c|c|}
\hline \multirow[b]{2}{*}{ Component } & \multirow{2}{*}{$\begin{array}{l}\text { Photon } \\
\text { energy }\end{array}$} & \multicolumn{2}{|c|}{$\mathrm{Au}_{100} \mathrm{Pt}_{0}$} & \multicolumn{2}{|c|}{$\mathrm{Au}_{90} \mathrm{Pt}_{10}$} & \multicolumn{2}{|c|}{$\mathrm{Au}_{80} \mathrm{Pt}_{20}$} & \multicolumn{2}{|c|}{$\mathrm{Au}_{70} \mathrm{Pt}_{30}$} & \multirow{2}{*}{$\begin{array}{l}\text { FWHM } \\
\text { (linked) }\end{array}$} \\
\hline & & B.E. & At $\%$ & B.E. & At $\%$ & B.E. & At $\%$ & B.E. & At $\%$ & \\
\hline \multirow{2}{*}{ Aubulk } & $220 \mathrm{eV}$ & 83.98 & 71 & 84.03 & 49 & 84.02 & 34 & 83.97 & 24 & 0.44 \\
\hline & $700 \mathrm{eV}$ & 84.10 & 72 & 84.10 & 57 & 84.12 & 47 & 84.02 & 35 & 0.66 \\
\hline \multirow{2}{*}{$A u_{\text {surf }}$} & $220 \mathrm{eV}$ & 83.85 & 29 & 83.74 & 29 & 83.68 & 18 & 83.63 & 12 & 0.54 \\
\hline & $700 \mathrm{eV}$ & 83.76 & 28 & 83.76 & 28 & 83.81 & 22 & 83.65 & 16 & 0.58 \\
\hline \multirow{2}{*}{$\mathrm{Pt}_{\mathrm{ox}}$} & $220 \mathrm{eV}$ & & & 73.17 & 3.6 & 72.79 & 5.2 & 73.35 & 3.5 & 2.3 \\
\hline & $700 \mathrm{eV}$ & & & 73 & 0.5 & 73 & 1.1 & 73 & 1.5 & 2.3 \\
\hline \multirow{2}{*}{ Ptbulk } & $220 \mathrm{eV}$ & & & 71.35 & 15 & 71.33 & 33 & 71.28 & 46 & 1.0 \\
\hline & $700 \mathrm{eV}$ & & & 71.30 & 12 & 71.35 & 24 & 71.20 & 38 & 1.2 \\
\hline \multirow{2}{*}{$\mathrm{Pt}_{\text {surf }}$} & $220 \mathrm{eV}$ & & & 70.66 & 3.9 & 70.69 & 9.4 & 70.73 & 14 & 0.59 \\
\hline & $700 \mathrm{eV}$ & & & 70.64 & 2.3 & 70.75 & 5.9 & 70.71 & 9.7 & 0.67 \\
\hline
\end{tabular}




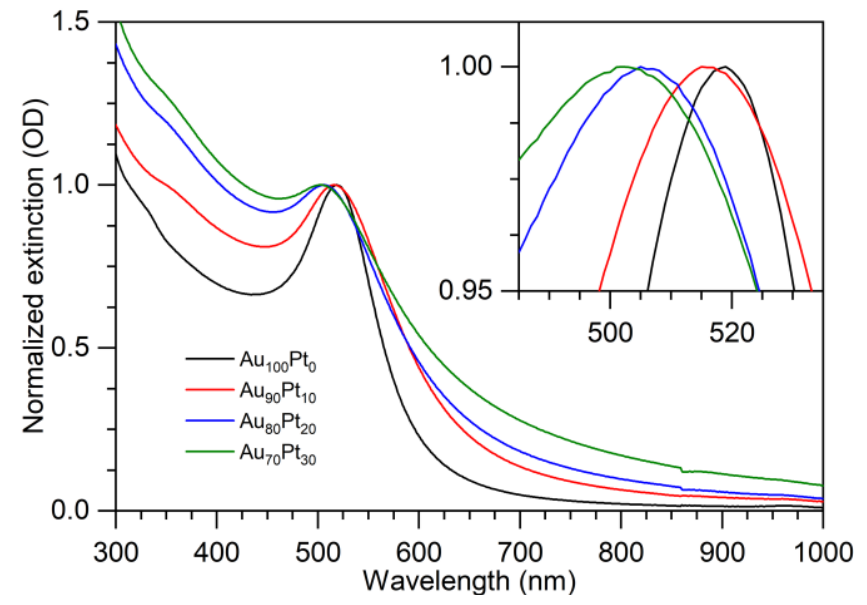

Figure S6. Steady-state optical extinction spectra of purified dispersions of Au and Au @Pt NCs normalized to 1.0 at their LSPR peak maxima. The inset shows a magnified view of the LSPR peaks.

Table S2. Fitting parameters from fits of Eq. 6 to the Au and Au@Pt NC extinction spectra presented in Figure 4.

\begin{tabular}{c|ccc|ccc|cccc}
\multicolumn{3}{c}{ LSPR } & \multicolumn{3}{c}{ vis IT } \\
Sample & $E_{0}(\mathrm{eV})$ & $\gamma_{0}(\mathrm{eV})$ & $c_{0}(\mathrm{OD})$ & $E_{1}(\mathrm{eV})$ & $\gamma_{1}(\mathrm{eV})$ & $c_{1}(\mathrm{OD})$ & $E_{2}(\mathrm{eV})$ & $\gamma_{2}(\mathrm{eV})$ & $c_{2}(\mathrm{OD})$ \\
\hline $\mathrm{Au}_{100} \mathrm{Pt}_{0}$ & 2.37 & 0.410 & 0.709 & 2.40 & 0.143 & 0.988 & 3.36 & 0.140 & 0.300 \\
$\mathrm{Au}_{90} \mathrm{Pt}_{10}$ & 2.38 & 0.650 & 0.697 & 2.40 & 0.244 & 0.985 & 3.36 & 0.140 & 0.300 \\
$\mathrm{Au}_{80} \mathrm{Pt}_{20}$ & 2.38 & 0.732 & 0.627 & 2.40 & 0.271 & 0.994 & 3.36 & 0.140 & 0.300 \\
$\mathrm{Au}_{70} \mathrm{Pt}_{30}$ & 2.37 & 0.841 & 0.664 & 2.40 & 0.309 & 0.985 & 3.36 & 0.140 & 0.300 \\
\hline
\end{tabular}

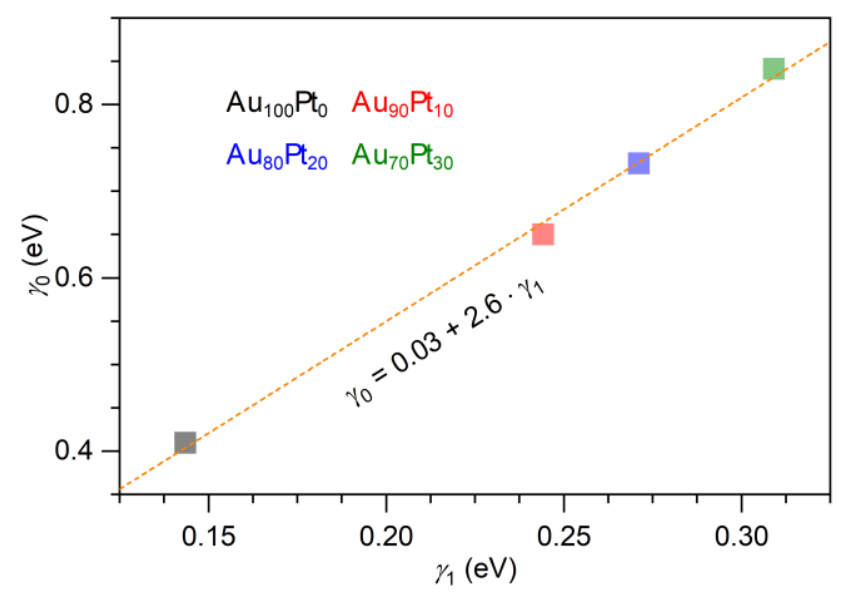

Figure S7. Correlation between the observed broadening of the LSPR and visible IT with increased Pt loading. 

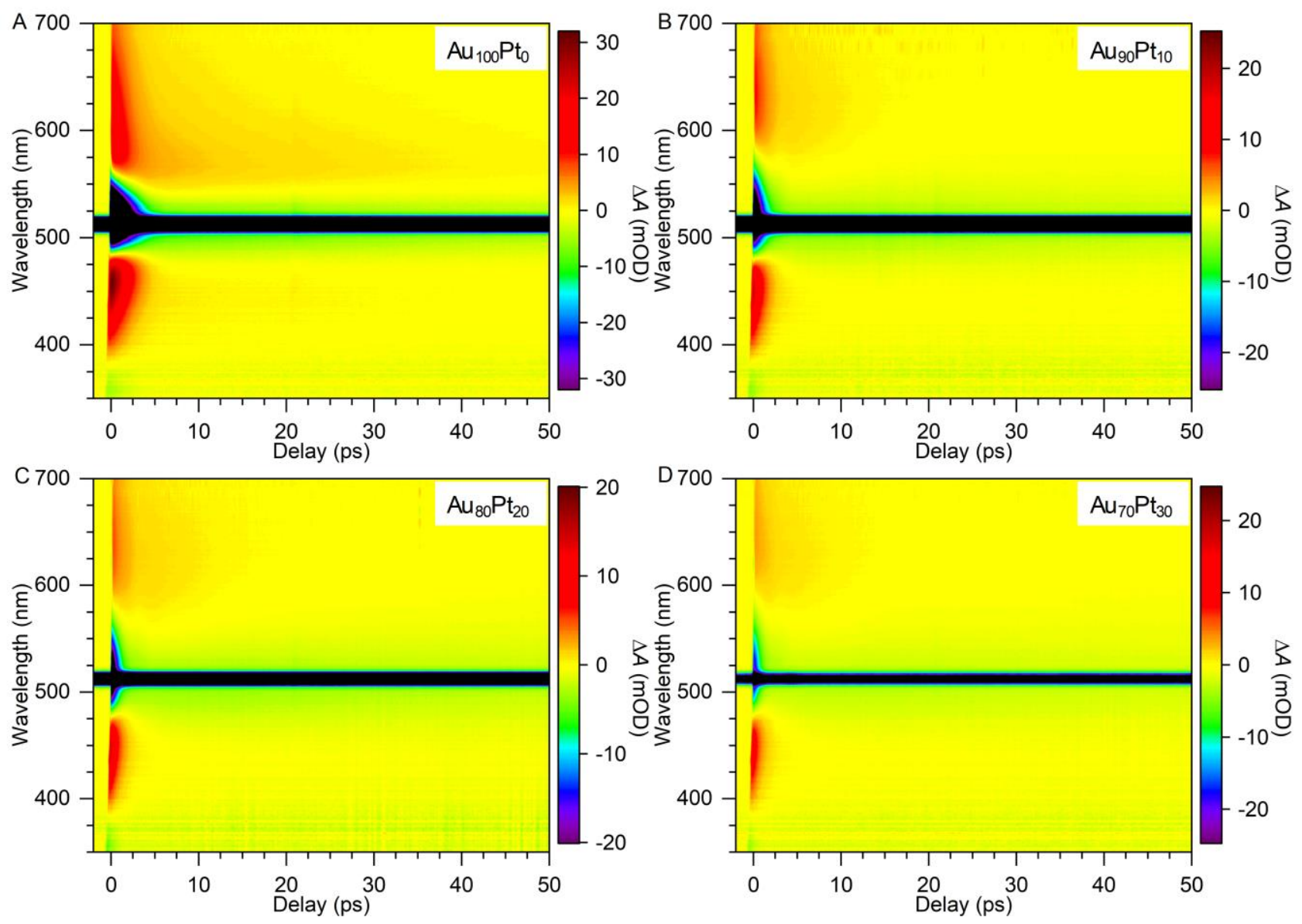

Figure S8. Raw transient absorption data of (A) $\mathrm{Au}_{100} \mathrm{Pt}_{0}$, (B) $\mathrm{Au}_{90} \mathrm{Pt}_{10},(\mathrm{C}) \mathrm{Au}_{80} \mathrm{Pt}_{20}$, and (D) $\mathrm{Au}_{70} \mathrm{Pt}_{30} \mathrm{NCs}$. Excited state absorption and bleach are indicated with red and blue colors, respectively. The color scale is normalized to the measured extinction at the pump energy. 


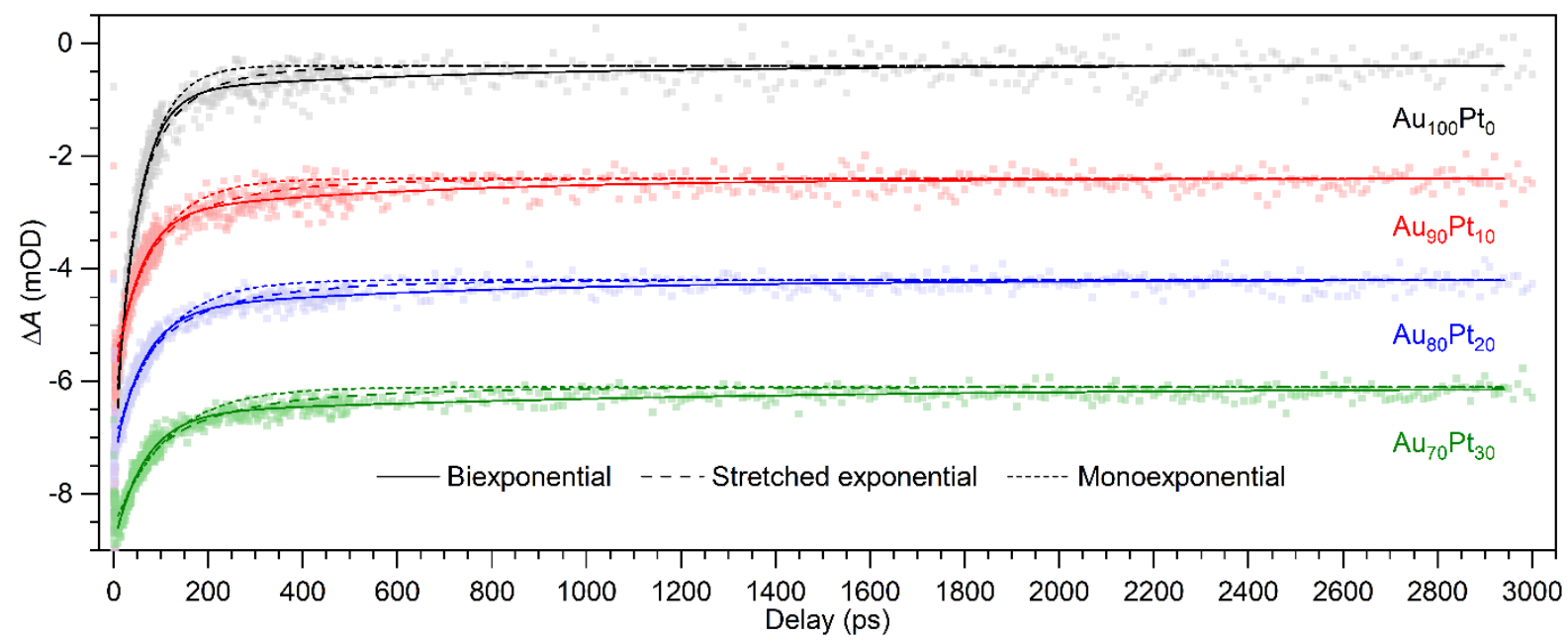

Figure S9. Slow dynamics observed at probe energy $=2.5 \mathrm{eV}(496 \mathrm{~nm})$. Three different fitting functions are shown: biexponential (solid), stretched exponential (dashed), and single exponential (short dashed). A stretching parameter $(\beta)$ of 0.7 was used for the stretched exponential fits. ${ }^{64}$ The data have been offset for clarity. 


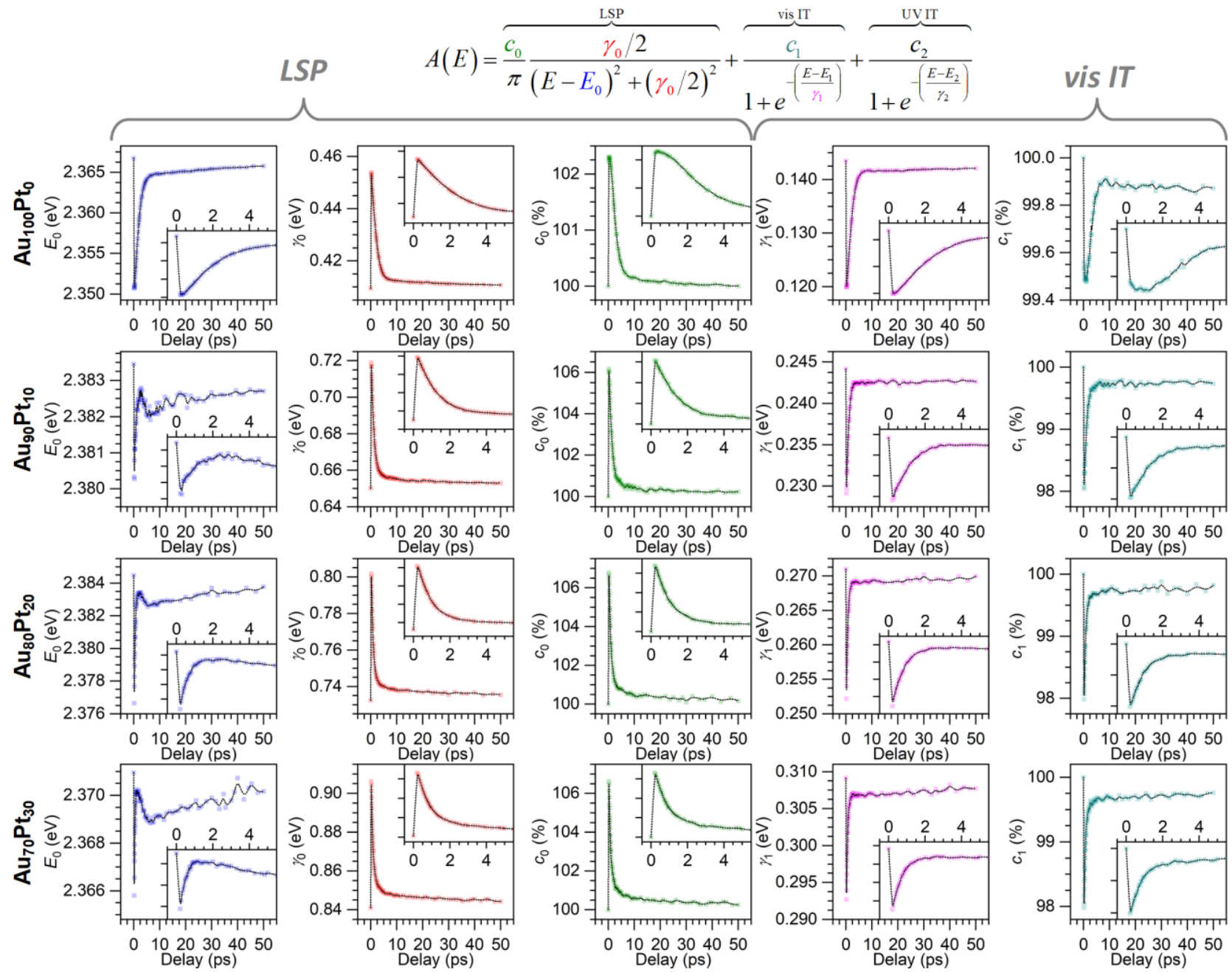

Figure S10. Time-dependence of all fitting parameters in the delay time range of 0-50 ps. Each row represents a sample. From top to bottom: $\mathrm{Au}_{100} \mathrm{Pt}_{0}, \mathrm{Au}_{90} \mathrm{Pt}_{10}, \mathrm{Au}_{80} \mathrm{Pt}_{20}$ and $\mathrm{Au}_{70} \mathrm{Pt}_{30} \mathrm{NCs}_{\text {. }}$ The symbol color represents a parameter in the expression shown at the top (identical to Eq. 2 in the text). From left to right, the panels depict LSPR peak energy (blue), LSPR width (red), LSPR amplitude (green), vis IT width (magenta), and vis IT amplitude (cyan). Insets show magnified views of the initial 0-5 ps. 


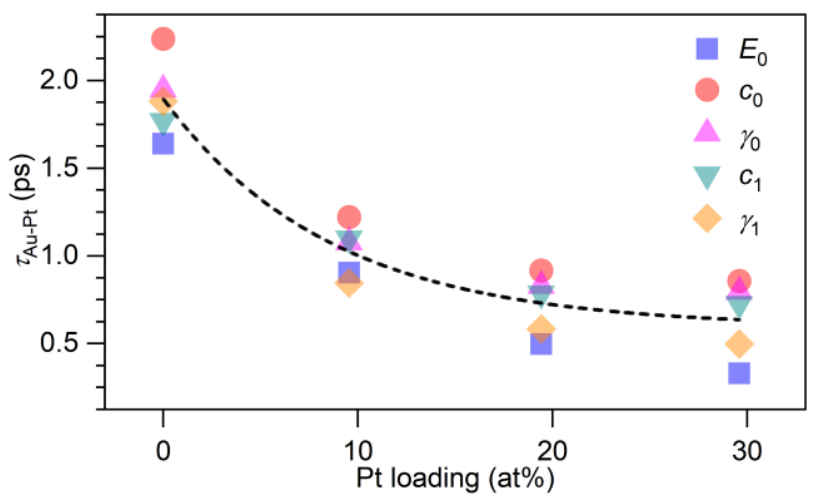

Figure S11. $\tau_{\mathrm{Au}-\mathrm{Pt}}$ as a function of Pt loading ( $\tau_{\mathrm{e}-\mathrm{p}}$ for Au NCs, i.e. 0 at $\% \mathrm{Pt}$ ) extracted from the time dependence of different fitting parameters of Eq. 6 (symbols). The dashed line represents an exponential fit to the average of $E_{0}, c_{0}, \gamma_{0}, c_{1}$ and $\gamma_{1}$. The average values are presented in Table 1. 